\title{
COMMENTS ON ANISOTROPIC YIELD CRITERIA
}

\author{
W. F. HOSFORD \\ Department of Materials and Metallurgical Engineering. University of Michigan. Ann Arbor, \\ MI 48109, U.S.A.
}

(Received 25 July 1984)

\begin{abstract}
Summary - Limitations of several anisotropic yield criteria are discussed. Hill's 1948 criterion overestimates the variation of yield strength with direction, while the specializations of Hill's 1979 theory assume planar isotropy. Shear stress terms cannot simply be included in either the author's 1979 criterion or in Hill's 1979 general criteria.

It is suggested that the author's criterion be modified so that it is expressed in terms of principal stresses rather than referring stresses to the symmetry axes.
\end{abstract}

\section{NOTATION}

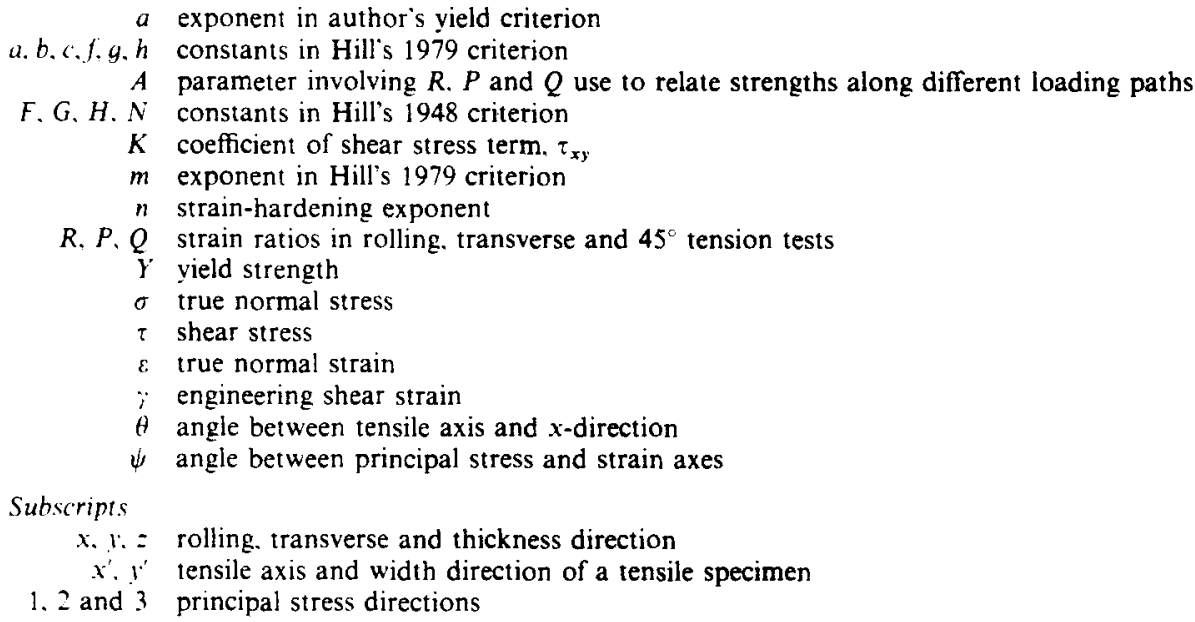

\section{INTRODUCTION}

For analyses of sheet-metal forming, an anisotropic yield criterion must be assumed. To be useful for engineering purposes it must reasonably describe the material behavior but be simple enough so its parameters can be easily evaluated, preferably by uniaxial tension tests. The purpose of this paper is to point out limitations of several anisotropic yield criteria and suggest a modification of one of them. The yield criteria considered are Hill's 1948 criterion [1]. Hill's 1979 generalized criterion with his four specializations [2] and a criterion suggested by the author in 1979 [3].

\section{YIELD CRITERIA}

The arguments which follow are addressed to the loading of sheet metals and therefore out-of-plane shear terms are neglected.

\section{Hill's 1948 criterion}

This may be written as:

$$
F\left(\sigma_{y}-\sigma_{z}\right)^{2}+G\left(\sigma_{z}-\sigma_{x}\right)^{2}+H\left(\sigma_{x}-\sigma_{y}\right)^{2}+2 N \tau_{x y}^{2}=\bar{\sigma}^{2} .
$$

With the corresponding flow rules the constants may be expressed in terms of strain-ratios measured in the $x, y$ and 45 directions.

$$
P \sigma_{x}^{2}+R \sigma_{y}^{2}+R P\left(\sigma_{x}-\sigma_{y}\right)^{2}+(2 Q+1)(R+P) \tau_{x y}^{2}=P(R+1) Y_{x}^{2},
$$

where $R=R_{0}, Q=R_{45}, P=R_{90}$ and $Y_{x}$ is the flow stress in an $x$-direction tension test. This is the only criterion which includes shear-stress terms. 
Hill's 1979 criterion

In its general form this is written as:

$f\left|\sigma_{2}-\sigma_{3}\right|^{m}+g\left|\sigma_{3}-\sigma_{1}\right|^{m}+h\left|\sigma_{1}-\sigma_{2}\right|^{m}+a\left|2 \sigma_{1}-\sigma_{2}-\sigma_{3}\right|^{m}+b\left|2 \sigma_{2}-\sigma_{3}-\sigma_{1}\right|^{m}+c\left|2 \sigma_{3}-\sigma_{1}-\sigma_{2}\right|^{m}=\bar{\sigma}^{m}$,

where the constants $f, g, h, a, b, c$ and $m$ are to be evaluated with various tests. Hill considered four special cases with $\sigma_{3}=0$ :

(1) $a=b=h=0, f=g$ so

$$
c\left|\sigma_{1}+\sigma_{2}\right|^{m}+f\left(\left|\sigma_{1}\right|^{m}+\left|\sigma_{2}\right|^{m}\right)=\bar{\sigma}^{m}
$$

(2) $a=b, c=f=g=0$ so

$$
a\left(\left|2 \sigma_{1}-\sigma_{2}\right|^{m}+\left|2 \sigma_{2}-\sigma_{1}\right|^{m}\right)+h\left|\sigma_{1}-\sigma_{2}\right|^{m}=\bar{\sigma}^{m}
$$

(3) $a=b, f=g, c=h=0$ so

$$
a\left(\left|2 \sigma_{1}-\sigma_{2}\right|^{m}+\left|2 \sigma_{2}-\sigma_{1}\right|^{m}\right)+f\left(\left|\sigma_{1}\right|^{m}+\left|\sigma_{2}\right|^{m}\right)=\bar{\sigma}^{m}
$$

(4) $a=b=f=g=0$ so

$$
c\left|\sigma_{1}+\sigma_{2}\right|^{m}+h\left|\sigma_{1}-\sigma_{2}\right|^{m}=\bar{\sigma}^{m}
$$

The general form recognizes the possibility of planar anisotropy, but since the 1,2 and 3 axes are the principal axes of anisotropy, it cannot be used for loading conditions which involve shear relative to these axes. In the four special forms $a=b$ and $f=g$ so planar isotropy is assumed. With planar isotropy the 1 and 2 axes may be oriented in any direction in the sheet so shear-stress terms are not necessary.

Author's 1979 criterion

This is also a special case of Hill's 1979 criterion in which $a=b=c=0$, so

which may be rewritten as

$$
\begin{gathered}
F\left|\sigma_{y}-\sigma_{z}\right|^{a}+G\left|\sigma_{z}-\sigma_{x}^{a}+H\right| \sigma_{x}-\left.\sigma_{y}\right|^{a}=\bar{\sigma}^{a}, \\
P \sigma_{x}^{a}+R \sigma_{y}^{a}+R P\left(\sigma_{x}-\sigma_{y}\right)^{a}=P(R+1) Y_{0}^{a}
\end{gathered}
$$

if $a$ is an even integer. Upper-bound calculations of yield loci for rotationally symmetric textures in fcc and bcc metals suggest that the best exponent, $a$, is 6 or 8. (3) This criterion does recognize planar anisotropy but is restricted in its present form to loading with principal stress axes coinciding with the principal axes of anisotropy.

\section{VARIATION OF YIELD STRENGTH WITH DIRECTION}

Hill's 1948 theory predicts:

$$
\frac{Y_{\theta}}{Y_{x}}=\left[\frac{P(R+1)}{(2 Q+1)(R+P) \cos ^{2} \theta \sin ^{2} \theta+R P\left(\cos ^{2} \theta-\sin ^{2} \theta\right)^{2}+P \cos ^{4} \theta+R \sin ^{4} \theta}\right]^{\frac{1}{2}} .
$$

For $\theta=90^{\circ}$ and $45^{\circ}$, this simplifies to

and

$$
\begin{aligned}
& Y_{90} / Y_{0}=[P(R+1) / R(P+1)]^{\frac{1}{2}} \\
& Y_{45} / Y_{0}=[2 P(R+1) /(Q+1)(R+P)]^{\frac{1}{t}}
\end{aligned}
$$

For a material which obeys power law work-hardening, $\bar{\sigma}=K \bar{\varepsilon}^{n}$, the ratio of tensile flow stresses in different directions at the same strain is given by ref. [6]

$$
\sigma_{\theta} / \sigma_{0}=A^{n+1},
$$

where $A$ is the right-hand side of equation (10), (11) or (12).

The author's criterion

Attempts to generalize equation (9) to include a shear-stress term $\tau_{x y}^{a}$ leads to an unreasonable criterion as shown below. By considering a $45^{\circ}$ tension test it can be shown that the coefficient. $K$, of the $\tau_{x x}^{a}$ term would be $(2 Q+1)(R$ $+P$ ) (see Appendix 1), so the criterion would be written as

$$
(2 Q+1)(R+P) \tau_{x y}^{a}+P \sigma_{x}^{a}+R \sigma_{y}^{a}+R P\left(\sigma_{x}-\sigma_{y}\right)^{a}=P(R+1) Y_{0}^{a} .
$$

If a $45^{\circ}$ tension test $\left(\sigma_{x}=\sigma_{y}=\tau_{x y}=Y_{45} / 2\right)$ is reconsidered, the predicted flow stress would be

$$
Y_{45}=2\left[\frac{P(R+1)}{2(Q+1)(R+P)}\right]^{1 / a} Y_{0} .
$$

If this is applied to a material with planar isotropy $\left(R=P=Q\right.$ and $\left.Y_{45}=Y_{x}\right)$ it reduces to $4^{1: a}=2$. so the exponent $a$ would have to be equal to 2 , and equation (14) would reduce to Hill's 1948 criterion (equation 1). Since equation (9) was proposed with exponents much larger than 2 to account for deviations from Hill's 1948 criterion, this is not a satisfactory conclusion. It should be noted that similar difficulties will arise if shear terms are introduced into Hill's 1979 general criterion, equation (3).

\section{NEW APPROACH}

A simple alternative to including shear-stress terms in an anisotropic criterion is to abandon the convention of expressing the stress components along the symmetry axes (i.e. 'principal axes of anisotropy') and assume that principal stress and principal strain axes coincide, whether or not they are parallel to the symmetry axes. It is 
recognised that this assumption may introduce errors, but these are likely to be much less than the errors that arise from assuming planar isotropy. Using this assumption the 1 and 2 axes are the axes of principal stress, so the author's criterion can be modified to:

$$
R_{2} \sigma_{1}^{a}+R_{1} \sigma_{2}^{a}+R_{1} R_{2}\left(\sigma_{1}-\sigma_{2}\right)^{a}=R_{2}\left(R_{1}+1\right) Y_{1}^{a} .
$$

where $R_{1}$ and $R_{2}$ are the strain ratios measured in the 1 and 2 directions, and $Y_{1}$ is the tensile yield strength in the 1 . direction. The corresponding flow rules are:

$$
\begin{aligned}
& \mathrm{d} \varepsilon_{1}: \mathrm{d} \varepsilon_{2}: \mathrm{d} \varepsilon_{3}= \\
& R_{2} \sigma_{1}^{a-1}+R_{1} R_{2}\left(\sigma_{1}-\sigma_{2}\right)^{a-1}: \\
& R_{1} \sigma_{2}^{a-1}+R_{1} R_{2}\left(\sigma_{2}-\sigma_{1}\right)^{a-1}: \\
& -R_{2} \sigma_{1}^{a-1}-R_{2} \sigma_{2}^{a-1} .
\end{aligned}
$$

The strength under balanced biaxial tension $\left(\sigma_{1}=\sigma_{2}\right)$ must be independent of the orientation of the axes. In this case

$$
\sigma_{1}=\left[\frac{R_{2}\left(R_{1}+1\right)}{R_{1}+R_{2}}\right]^{1 / a} Y_{1}=\text { constant }
$$

substituting $\left(R_{1}=R, R_{2}=P, Y_{1}=Y_{x}\right)$ and $\left(R_{1}=R_{\theta}, R_{2}=R_{\theta+90}, Y_{1}=Y_{\theta}\right)$ into equation (17), the variation of tensile flow stress with direction can be written as

$$
\frac{Y_{\theta}}{Y_{x}}=\left[\frac{P(R+1)\left(R_{\theta}+R_{\theta+90}\right)}{(R+P) R_{\theta+90}\left(R_{\theta}+1\right)}\right]^{1 / a} .
$$

For the 90 and $45^{\circ}$ cases this reduces to

and

$$
\frac{Y_{y}}{Y_{x}}=\left[\frac{P(R+1)}{R(P+1)}\right]^{1 / a}
$$

$$
\frac{Y_{45}}{Y_{x}}=\left[\frac{2 P(R+1):}{(R+P)(Q+1)}\right]^{1 / 0}
$$

which are identical to equations (11) and (12) except for the exponent.

Again equation (13i can be used to compare tensile flow stresses at the same strain level. Now

$$
\begin{aligned}
& \frac{\sigma_{90}}{\sigma_{0}}=\left[\frac{P(R+1)}{R(P+1)}\right]^{n+1 / a} \\
& \frac{\sigma_{45}}{\sigma_{0}}=\left[\frac{2 P(R+1)}{(R+P)(Q+1)}\right]^{n+1 / a} .
\end{aligned}
$$

In Fig. 1. experimental results from tension tests on various steels at 0.45 and $90^{\circ}$ [4-8] are compared with equations (21) and (22). Reported ratios of $\sigma_{90} / \sigma_{0}$ and $\sigma_{45} / \sigma_{0}$ are plotted against

$$
\left[\frac{P(R+1)}{R(P+1)}\right]^{n+1} \text { and }\left[\frac{2 P(R+1)}{(R+P)(Q+1)}\right]^{n+1} \text {, }
$$

respectively. It is clear that $a=8$ represents the data much better than $a=2$ (Hills 1948 criterion).

\section{DEVIATION OF PRINCIPAL STRESS AND STRAIN AXES}

Only Hill's (1948) theory can quantitatively predict how much the principal stress and strain axes may diverge. Consider a tension test along an axis. $x^{\prime}$, oriented at an angle $\theta$ to $x$ and let $y^{\prime}$ be the width direction of the specimen (Fig. 2). Using the flow rules associated with Hill's 1948 criterion the ratio of shear strain $d \gamma_{x^{\prime}} y^{\prime}$ to $d \varepsilon_{x^{\prime}}-\mathrm{d} \varepsilon_{y^{\prime}}$ is shown (see Appendix 2) to be:

$$
\frac{\mathrm{d}_{i x^{\prime} y^{\prime}}}{\mathrm{d} \varepsilon_{x^{\prime}}-\mathrm{d} \varepsilon_{y^{\prime}}}=\frac{(K-4 R P-2 P) \cos ^{3} \theta \sin \theta-(K-4 R P-2 R) \sin ^{3} \theta \cos \theta}{P(2 R+1) \cos ^{4} \theta+R(2 P+1) \sin ^{4} \theta+(2 K-4 R P-R-P) \cos ^{2} \theta \sin ^{2} \theta},
$$

where $K=(2 Q+1)(R+P)$. The Mohr's circle relations can be used to find the angle, $\psi$, between the principal stress and strain axes:

$$
\tan (2 \psi)=\frac{\mathrm{d} \gamma_{x y}}{\mathrm{~d} \varepsilon_{x^{\prime}}-\mathrm{d} \varepsilon_{y^{\prime}}}
$$

Unless there are extreme differences between $R, Q$ and $P$, the predicted values of $\psi$ are small; Table 1 shows the extreme predicted values of $\psi$ for several materials and the corresponding values of $\theta$.

To check the predictions of equations (23) and (24), tensile tests were made on four duplicate specimens of the dual phase steel cut at $\theta=25^{\circ}$, and four specimens of the brass cut at $\theta=22^{\circ}$. Each specimen was gridded prior to testing and measurements of the grids before and after testing were used to make four measurements of $\psi$ on each specimen. The measured values of $\psi$ were $-1.15 \pm 0.47^{\circ}$ for the dual-phase steel and $+1.99^{\circ} \pm 0.37^{\circ}$ for the brass where the \pm 0.47 and \pm 0.37 represent the standard deviations. These measured values are significantly lower than the values of $\psi$ predicted from equations (23) and $(24)\left(-4.08^{\circ}\right.$ for the dual-phase steel and $+3.56^{\circ}$ for the brass). Since the tensile 


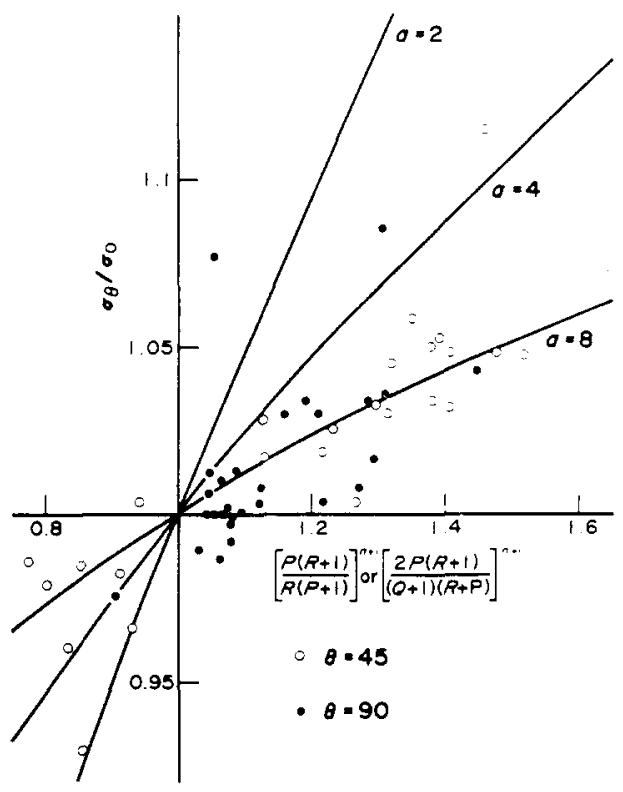

Fig. 1. Experimental ratios of flow stress $\sigma_{90} / \sigma_{0}$ and $\sigma_{45} / \sigma_{0}$ compared with parameters for predicting these ratios. Data are for various grades of steel (ref.[4-8]).

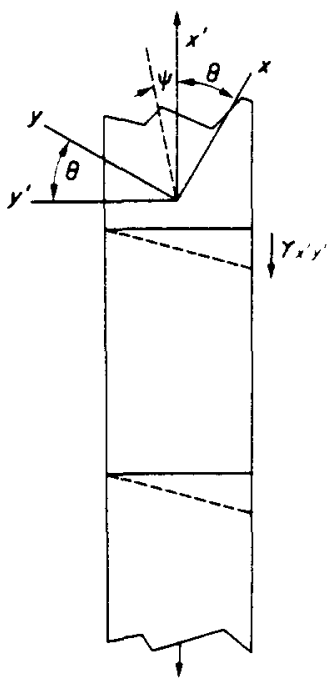

FIG. 2. Schematic illustration of tensile specimen. Equation (23) predicts a finite shear strain for nonsymmetric orientations. $\psi$ is the angle between the principal stress axis, $x^{\prime}$, and the principal strain axis.

Table 1. Predicted Shear Strains

\begin{tabular}{lccccc}
\hline Material & $R$ & $P$ & $Q$ & $\theta^{*}$ & $\psi^{*}$ \\
\hline Dual-phase steel & 1.15 & 1.25 & 0.80 & $25^{\circ}$ & $-4.08^{\circ}$ \\
Annealed brass & 0.81 & 0.67 & 1.00 & $22^{\circ}$ & $+3.56^{\circ}$ \\
A K steel & 1.90 & 2.32 & 1.52 & $25^{\circ}$ & -3.96 \\
6062 aluminum & 0.67 & 0.75 & 0.51 & $25^{\circ}$ & -3.06 \\
\hline
\end{tabular}

*For maximum value of $|\psi|$. 
specimens were cut at angles for which the maximum values of $\psi$ were predicted, these preliminary results suggest that the assumption that principal axes of stress and strain coincide should not lead to large errors.

\section{LIMITATION}

The proposed formalism provides no way of predicting the necessary parameters, $R_{\theta}$, from measurements of $R, Q$ and $P$ as does Hill's 1948 theory. Hill's 1948 criterion does give a reasonable approximation to the variation of $R_{\theta}$ with $\theta$, and at this time no alternative is proposed.

\section{CONCLUSIONS}

It is suggested that for in-plane loading equation (15) with $a=6$ or 8 may give the best approximation for anisotropic metals. To apply this criterion it is further suggested that the angular variation of $R_{\theta}$ be found using Hill's 1948 criterion together with experimental values of $R, P$ and $Q$.

Acknowledgements-The author wishes to thank R. M. Caddell and R. W. Logan for stimulating discussions.

\section{REFERENCES}

1. R. Hill, Proc. R. Soc. Lond. Ser. A 193, 281 (1948).

2. R. Hill, Math. Proc. Camb. Soc. 75, 179 (1979).

3. W. F. Hosford, Proc. 7th North Am. Metalworking Conf. S.M.E., Dearborn, Michigan, U.S.A. 191 (1979). See also R. W. Logan and W. F. Hosford, Int. J. Mech. Sci. 22, 419 (1980).

4. A. N. Bramley and P. B. Mellor, Int. J. Mech. Sci. 8, 101 (1966).

5. I. L. Dillamore, R. J. Hazel, T. W. Watson and P. Hadden, Int. J. Mech. Sci. 13, 1049 (1971).

6. C. Vial, W. F. Hosford and R. M. CADdell, Int. J. Mech. Sci. 25, 419 (1983).

7. G.-C. JUN and W. F. Hosford (Submitted for publication).

8. D. Meuleman, Ph.D. Thesis, University of Michigan, U.S.A. (1980).

\section{APPENDIX 1}

Assume a criterion of the form

$$
K \tau_{x y}^{a}+P \sigma_{x}^{a}+R \sigma_{y}^{a}+R P\left(\sigma_{x}-\sigma_{y}\right)^{a}=a f .
$$

The corresponding flow rules would be

$$
\begin{gathered}
\mathrm{d} \varepsilon_{x}: \mathrm{d} \varepsilon_{y}: \mathrm{d} \varepsilon_{x}: \mathrm{d} \gamma_{x y}= \\
P \sigma_{x}^{a-1}+R P\left(\sigma_{x}-\sigma_{y}\right)^{a-1}: R \sigma_{y}^{a-1}+R P\left(\sigma_{y}-\sigma_{x}\right)^{a-1}:-R \sigma_{x}^{\alpha-1}-R \sigma_{y}^{\alpha-1}: K_{x_{x y}}^{a-1}
\end{gathered}
$$

Consider a tension test along $x^{\prime}$ oriented at $45^{\circ}$ to $x$ and $y$. At yielding

$$
\begin{aligned}
\sigma_{x}=\sigma_{y}=\tau_{x y} & =\frac{1}{2} Y_{45} \\
\mathrm{~d} \varepsilon_{x}: \mathrm{d} \varepsilon_{y}: \mathrm{d} \varepsilon_{z}: \mathrm{d} \gamma_{x y} & =P: R:-(R+P): K .
\end{aligned}
$$

The transverse strain $\mathrm{d} \varepsilon_{y^{\prime}}$ would be

$$
\mathrm{d} \varepsilon_{y^{\prime}}=\frac{1}{2} \mathrm{~d} \varepsilon_{x}+\frac{1}{2} \mathrm{~d} \varepsilon_{y}-\frac{1}{2} \mathrm{~d} \gamma_{x y}
$$

so the value of $Q=R_{43}$

$$
Q=\frac{\mathrm{d} \varepsilon_{y}}{\mathrm{~d} \varepsilon_{z}}=\frac{(K-R-P) / 2}{R+P}
$$

Solving for $K$.

$$
\mathbf{\Lambda}-(2 Q+1)(R+P) \text {. }
$$

\section{APPENDIX 2}

For a tension test along $x^{\prime}$ the stresses will be $\sigma_{x}=\sigma_{x^{\prime}} \cos ^{2} \theta, \sigma_{y}=\sigma_{x^{\prime}} \sin ^{2} \theta$ and $\tau_{x y}=\sigma_{x^{\prime}} \cos \theta \sin \theta$. Equation (A-1) becomes

$$
\begin{aligned}
& \mathrm{d} \varepsilon_{x}: \mathrm{d} \varepsilon_{y}: \mathrm{d} \varepsilon_{z}: \mathrm{d} \gamma_{x y}= \\
& P \cos ^{2} \theta+R P\left(\cos ^{2} \theta-\sin ^{2} \theta\right): \\
& R \sin ^{2} \theta+R P\left(\sin ^{2} \theta-\cos ^{2} \theta\right): \\
& -P \cos 2 \theta-R \sin ^{2} \theta \\
& K \cos \theta \sin \theta .
\end{aligned}
$$

The tensile specimen will undergo strains

$$
\begin{aligned}
\mathrm{d} \gamma_{x^{\prime} y^{\prime}} & =-2 \mathrm{~d} \varepsilon_{x} \cos \theta \sin \theta+2 \mathrm{~d} \varepsilon_{y} \cos \theta \sin \theta+\mathrm{d} \gamma_{x y}\left(\cos ^{2} \theta-\sin ^{2} \theta\right) \\
\mathrm{d} \varepsilon_{x^{\prime}} & =\mathrm{d} \varepsilon_{x} \cos ^{2} \theta+\mathrm{d} \varepsilon_{y} \sin ^{2} \theta+\mathrm{d} \gamma_{x y} \cos \theta \sin \theta \text { and } \\
\mathrm{d} \varepsilon_{y^{\prime}} & =\mathrm{d} \varepsilon_{x} \sin ^{2} \theta+\mathrm{d} \varepsilon_{y} \cos ^{2} \theta-\mathrm{d} \gamma_{x y} \cos \theta \sin \theta .
\end{aligned}
$$

Combining equations (A-5) and (A-6) results in equation (23). 